\title{
Adjacent Channel Interference due to Wavelength Drift of a Tunable Laser in Base-Band and Subcarrier Multiplexed System
}

\author{
E. Connolly, A. Kaszubowska-Anandarajah and L.P. Barry \\ School of Electronic Engineering, Dublin City University, Dublin 9, IRELAND. \\ Phone: + 3531700 7635, Fax: + 3531700 5508, Email: aleksandra.kaszubowska@dcu.ie
}

\begin{abstract}
Summary
Wavelength Packet Switching (WPS) using fast Tunable Lasers (TLs) has gained a lot of interest in recent years. Many researchers consider it to be an enabling technology for the all optical networks. In WPS system the data is transmitted on a destination dependent wavelength. The routing and switching of the data could be realized on a packet-by-packet basis with the TL generating a required wavelength for each packet [1,2]. The characteristics of a tunable transmitter would have a strong influence on the performance of a WPS network. The switching time will determine the throughput of the system, Side Mode Suppression Ratio (SMSR) together with wavelength stability would influence the minimum channel spacing therefore limiting the capacity of the system. The latter parameter is especially important for the metro and access networks, which are migrating towards Dense Wavelength Division Multiplexing (DWDM). However, reduction in the channel spacing puts very stringent requirements on the performance of a TL. In this paper, we examine the amplitude and the duration of a wavelength drift of the TL at the most crucial moment, which is the time after the wavelength switch and measure the impact of this drift on the performance of the DWDM system spaced by $12.5 \mathrm{GHz}$. The adjacent channel interference is examined for two cases: firstly when the TL is modulated with Base-Band (BB) data, secondly when Subcarrier Multiplexing (SCM) is used. The experimental set-up for both cases are shown in Fig. 1 (a) and (b).
\end{abstract}
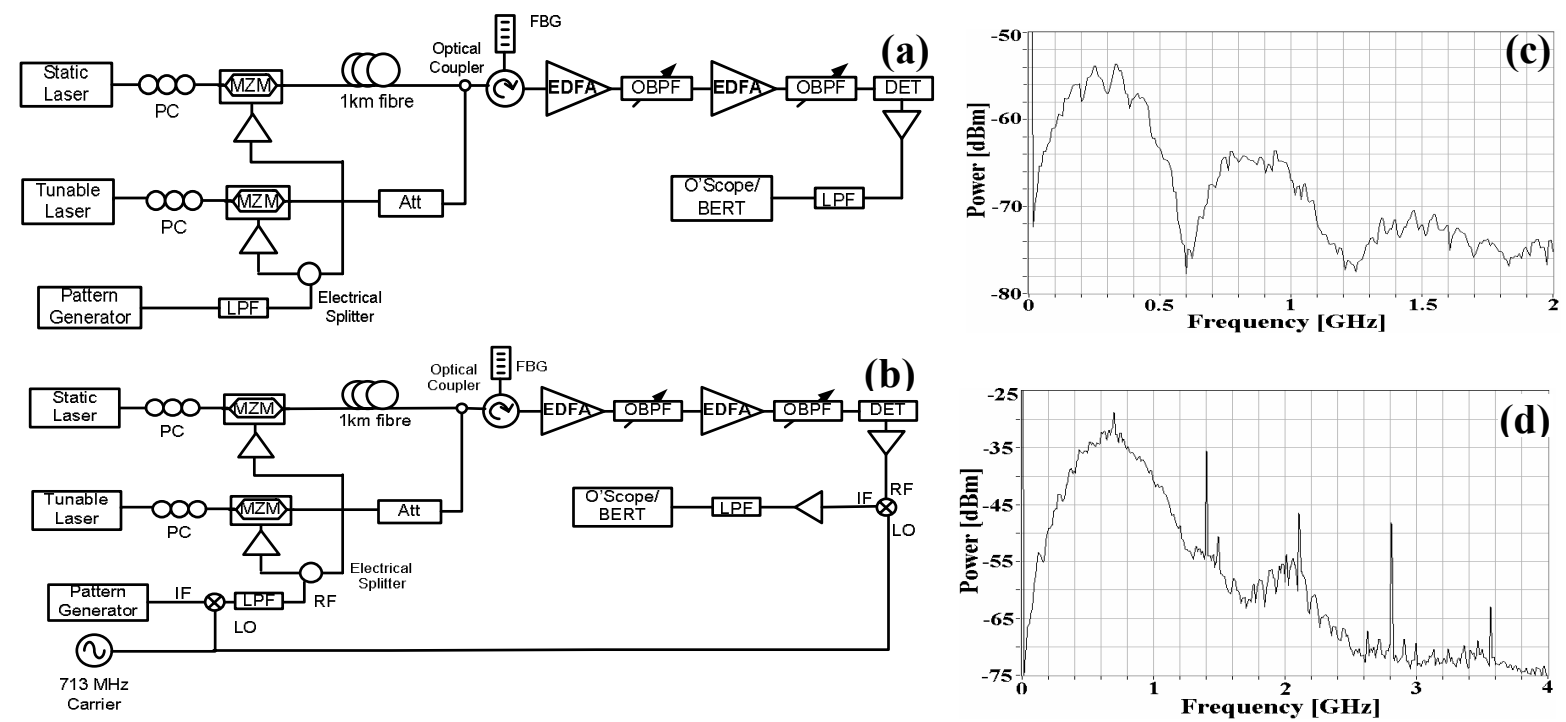

Fig. 1: Experimental set-up: (a) WPS using BB data, (b) WPS using SCM; electrical spectrum of (c) BB data, (d) SCM signal

The experimental set-up consists of two lasers: a TL at $1546.096 \mathrm{~nm}$ (channel 49) and a static laser at $1545.996 \mathrm{~nm}$ (12.5 GHz away). Both lasers are externally modulated in the first case (Fig. 1(a)) with $622 \mathrm{Mb} / \mathrm{s} \mathrm{BB}$ data, in the second (Fig. 1(b)) with $622 \mathrm{Mb} / \mathrm{s}$ data upconverted to $713 \mathrm{MHz}$. The BB data is passed through a $467 \mathrm{MHz}$ LowPass Filter (LPF), the SCM signal on the other hand is limited in its spectral width using a $757 \mathrm{MHz}$ LPF. The signals are then split and amplified using electrical amplifiers before being applied to two external modulators with a bandwidth of $2.5 \mathrm{~Gb} / \mathrm{s}$ each. Because the data used for both lasers was identical we needed to de-correlate it. In order to do so, the signal from the fixed laser was sent over a length of fiber before being combined with the output of the TL (attenuated to equalize the powers between both channels). At the receiver the static laser was filtered using a Fiber Bragg Grating (FBG) with a bandwidth of $4 \mathrm{GHz}$, optically pre-amplified and filtered (twice) and detected using a photodiode. After detection the signals were amplified subsequent to which the BB data was filtered using a LPF and fed directly to an error detector, while the SCM signal was downconverted using a mixer and a local oscillator, before being filtered and characterized. The electrical spectra of the modulating BB and SCM signals are shown in Fig. 1 (c) and (d) respectively. From the figures it can be seen that the BB signal has relatively 
strong (-16 dB below the main lobe) spectral components at around $1.5 \mathrm{GHz}$. For the SCM signal those can be found at around 2 and $2.8 \mathrm{GHz}$.

It should be noted that the TL used has a Semiconductor Optical Amplifier (SOA) placed at the output. When the TL switches between wavelengths this device is turned off momentarily in order to attenuate any spurious components generated during the wavelength tuning [3]. The duration of this blanking time is around $60 \mathrm{~ns}$ and after this time the TL is guaranteed to be within a range of $15 \mathrm{GHz}$ from its destination wavelength. Having characterized our laser we found out that for the transition between channel $13(1560.606 \mathrm{~nm})$ and 49 the wavelength drift of the TL is the largest and it is plotted as a function of time (from the beginning of the switch) in Fig. 2(a). From the plot it can be seen that the amplitude of the drift is around $8 \mathrm{GHz}$ [4].

In order to characterize the adjacent channel interference due to the wavelength drift of the TL we measure the Bit Error Rate (BER) of the static laser for three different modes of TL operation: (1) when TL is static at channel 13, (2) when TL is static at channel 49 (12.5 GHz away from the fixed laser) and when TL is switching between channel 13 and 49 for both the BB and the SCM data. The BER vs. received optical power plots for the BB and SCM data are shown in Fig. 2(b) and (c) respectively. It can be seen that, when the TL is switching, wavelength drift of the TL causes an adjacent channel interference, which places an error floor for both types of signals. The impact of this interference is much more severe for the BB data (BER of 10e-6) than for the SCM signal (BER 10e-8) even though the separation between the SCM signals is smaller than for the BB data. This difference in performance is due to the fact that in case of the BB any portion of the light from the TL that leaks through the OBPF, interferes with the data carried by the static laser. However, in case of SCM the side bands of the two lasers have to spectrally overlap (as a result of TL drift) in order to degrade the quality of the signal transmitted by the fixed laser. If the condition for spectral overlapping is not fulfilled, the light from the TL will beat at the detector with the optical carrier of the static laser to produce an RF signal at different frequency than the desired signal. This additional component can be then electrically filtered out using a LPF. However, in a BB system minimization of the interference due to TL wavelength drift would require the use of a very narrow optical filter. This solution though carriers the risk of losing the signal if its wavelength fluctuated and drifted out of the pass-band of the filter. Nonetheless, an optimal solution would be to use a wider OBPF in conjunction with SCM transmission. In this case the filtering of the interfering signals could be performed electrically (using a LPF), which is cheaper and independent on the wavelength drift of the demultiplexed signal itself.
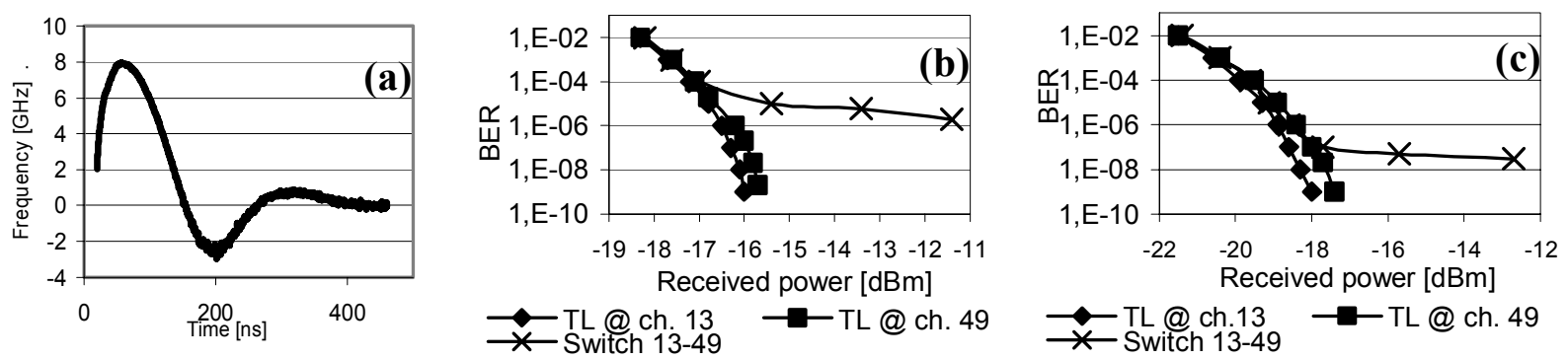

Fig. 2: (a) TL wavelength drift; BER vs. received optical power for (a) BB data, (b) SCM signal for different modes of operation of the TL

The impact of the adjacent channel interference due to wavelength drift of the TL has been characterized for a system transmitting either BB or SCM data. The BER measurements show that SCM is more resistant to this degrading factor allowing for a closer channel spacing. In case of SCM the interference from the TL drift degrades the quality of the signal only when the drift is large enough to cause the sidebands from the adjacent channel to overlap.

Acknowledgements: We wish to acknowledge the support of Science Foundation Ireland.

References

1. L. Ponnampalam et al. " Dynamically Controlled Channel-to-Channel Switching in a Full-Band DS-DBR Laser," IEEE J. Quantum Electron., vol. 42, pp. 223-230, 2006

2. I. White, et al.: Wavelength Switching Components for Future Photonic Networks, IEEE Comms. Mag., vol. 40. pp. 74-81, Sept. 2002

3. A. J. Ward et al. "Widely tunable DS-DBR laser with monolithically integrated SOA: design and performance," IEEE J. Sel. Topics Quantum Electron., vol. 11, no. 1, pp. 149-156, 2005

4. E. Connolly et al. "Cross Channel Interference due to Wavelength Drift of Tuneable Lasers in DWDM Networks," paper Mo. P.13, ICTON 2006, Nottingham, UK 